\title{
Electronic Textiles in Computer Science Education:
}

\section{A Synthesis of Efforts to Broaden Participation, Increase Interest, and Deepen Learning}

\author{
Gayithri Jayathirtha $^{\dagger}$ \\ Graduate School of Education \\ University of Pennsylvania \\ Philadelphia PA USA \\ gayithri@gse.upenn.edu
}

\author{
Yasmin B. Kafai \\ Graduate School of Education \\ University of Pennsylvania \\ Philadelphia PA USA \\ kafai@upenn.edu
}

\begin{abstract}
Just over a decade ago, various electronic textile construction kits have emerged with Arduino-based microcontrollers, sensors, and actuators that can be sewn together with conductive thread to create wearables augmented with new functionalities. These kits were designed to broaden participation in and perspectives about computing along with introducing learners to powerful ideas about circuitry and coding. In this paper, we synthesize 46 studies that have introduced crafts, circuitry, and coding concepts with e-textiles in K-16 education. We found that etextiles have been successful in broadening participation and increasing interest in computing for many youth and adults, especially from underrepresented groups, inside and outside of school. While the e-textiles construction kits have been successful in deepening learning of circuitry concepts, learning of computer science, especially advanced concepts, has been far less attended to with the exception of projects designed by undergraduate students with already significant disciplinary expertise. In the discussion, we suggest directions for future research such as bringing more computing into e-textile designs, creating assessments to capture coding and circuitry learning, and developing models for more advanced projects to leverage the potential of e-textiles for computer science education.
\end{abstract}

\section{CCS CONCEPTS}

- Applied computing $\rightarrow$ Education $\rightarrow$ Interactive Learning Environments

\section{KEYWORDS}

Electronic textiles, computational construction kits, computer science education, gender and diversity

\section{${ }^{\dagger}$ Corresponding Author}

Permission to make digital or hard copies of all or part of this work for personal or classroom use is granted without fee provided that copies are not made or distributed for profit or commercial advantage and that copies bear this notice and the full citation on the first page. Copyrights for components of this work owned by others than ACM must be honored. Abstracting with credit is permitted. To copy otherwise, or republish, to post on servers or to redistribute to lists, requires prior specific permission and/or a fee. Request permissions from Permissions@acm.org.

SIGCSE'19, February 27-March 2, 2019, Minneapolis, MN, USA.

(C) 2019 Association of Computing Machinery.

ACM ISBN 978-1-4503-5890-3/19/02 ..\$15.00.

DOI: https://doi.org/10.1145/3287324.32873430

\section{ACM Reference format:}

Gayithri Jayathirtha and Yasmin Kafai. 2019. Electronic Textiles in Computer Science Education: A Synthesis of Efforts to Broaden Participation, Increase Interest, and Deepen Learning. In Proceedings of the 50th ACM Technical Symposium on Computer Science Education (SIGCSE '19). Feb. 27-Mar. 2, 2019, Minneapolis, MN, USA. ACM, New York, NY, USA. 7 pages. https://doi.org/10.1145/3287324.3287343

\section{Introduction}

To address the underrepresentation of women and minorities in the tech industry [1], many efforts are underway to broaden participation in K-12 computer science (CS) education, motivate interest in computing and not only introduce but also deepen their understanding of computational ideas. Many outreach efforts such as Code Start, Girls Who Code, and Black Girls Code (for example, [2]) and community spaces such as Computer Clubhouses [3] have provided opportunities for underrepresented groups to learn computer programming. Programming tools such as Scratch [4], Alice [5] and others have been designed to provide a low threshold for exploring computational ideas and connect to a variety of interests. Further, high school curricula such as Exploring Computer Science [6] have been developed to introduce computing to students in under-resourced communities.

One aspect that has not received as much attention is the introduction of new tools, materials and practices for learning computer science which challenge traditional conceptions of computing. One such example are electronic textiles (hereafter: e-textiles) that connect computing and crafting-two activities not commonly associated with each other-combining traditionally masculine activities of computing and engineering with the more traditionally feminine activities of sewing and crafting [7]. E-textile construction kits provide electronic components such as LED lights, sensors, and microcontrollers with sewable interface that can be stitched on clothing, accessories, and toys using conductive thread, and usually programmed through the conventional Arduino IDE. Over the last decade, numerous e-textile construction kits have been developed [8]. Four of these-the LilyPad Arduino (Sparkfun), micro:bit (BBC), and Flora and the Circuit Playground (Adafruit) have moved into commercial production and distribution, making these kits broadly accessible and more affordable for K16 education. At the same time, dozens of studies have been 
conducted in which hundreds of learners of all ages and backgrounds have engaged with e-textiles inside and outside of school.

In this paper, we take stock of the overall contribution of etextiles to computer science education. The introduction of etextiles as an alternative to construction kits focused on robotics and gaming has been accompanied by hopes to broaden participation, increase interest in computing and further the learning of computing concepts. With many individual studies involving e-textiles completed, this comprehensive review will provide a more systematic and cumulative account on the trends in the field at large. Given the still exploratory nature of the research studies and the current state of computer science education [9], we conducted a meta-synthesis [10] rather than a meta-analysis which would be more suitable for fields with more statistical studies. Meta-syntheses have been common in public health and medical fields but are now finding more reception in educational contexts [11]. By searching through databases and consulting with experts in the field, we developed a repository of empirical studies on e-textiles to address the following research questions: (1) To what degree have e-textiles succeeded in broadening participation of underrepresented groups in computing? (2) To what degree have e-textiles raised students' interest in computing? and (3) What can students learn when making e-textile projects? In the discussion we address critical issues and outline steps for further research.

\section{Background}

The first e-textiles construction kit consisted of bulky, repurposed computer components connected together and attached to clothing to make a musical jacket, one that allowed the wearer to play music using the embroidered sections on its front [12]. Almost a decade later, the arrival of the Arduino board, a low-cost, open-source microcontroller led to the development of the LilyPad Arduino [7]. The LilyPad Arduino, prototyped by Buechley on a fabric substrate in a university research lab was later developed and distributed by Sparkfun (see Figure 1, left) as a printed circuit board with metal-coated holes, but with the same Arduino microcontroller and programmable input/output pins. Development of peripheral electronic components such as LED lights, temperature and light sensors, and switches soon followed these initial efforts. More recently, Adafruit Industries also developed similar boards such as Circuit Playground, this time with more on-board sensors such as accelerometer, temperature, and light sensors (see Figure 1, right), making it easier to realize interactive artifacts. Other commercially-available, simplified e-textile construction kits such as LilyTiny, Gemma and Flora provide pre-programmed functionalities or fewer input and output pins. In addition, researchers have developed other versions such as Teeboards [13] and Quilt Snaps [7, 14], but these have not gone into commercial distribution.
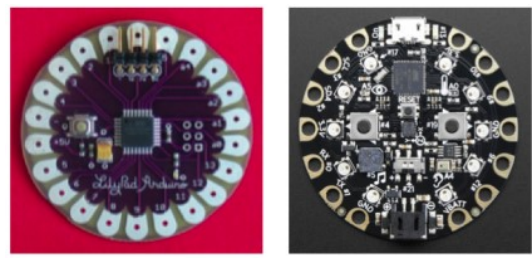

Figure 1: LilyPad Arduino board showing the sewable and electronic holes (left); Adafruit Circuit Playground board with many more on-board sensors (right)

One of the attractions for developing e-textiles for Buechley and colleagues was that "textile crafts might prove to be an effective means of promoting girls' enthusiasm toward programming and engineering" (p. 56) [14]. This aspect of broadening participation in computing was confirmed when Buechley and Hill [15] conducted a study of projects made with the Lilypad in "the wild" comparing them to typical Arduinobased projects. They studied the sales records of LilyPad and Arduino boards from the SparkFun site and analyzed the publicly-available project details to understand gender composition of the customers. Although a much smaller proportion of the overall customer base purchased LilyPad (12\%) compared to Arduino (91\%), they noted that only $2 \%$ of the Arduino customers were female while $35 \%$ of the LilyPad customers were female. Further, $65 \%$ of the LilyPad projects were developed by female makers, which is much higher than the small proportion $(2 \%)$ of the Arduino projects where the makers were female. Buechley and Hill's [15] findings revealed that indeed new tools such as LilyPad Arduino can create new computing communities. With e-textiles moving into K-16 education, one of our central questions was whether this trend of increasing gender diversity among participants in computation would continue. Furthermore, we sought to see if the innovative sewable interface of e-textiles kits broadened participation across dimensions other than gender to include learners from groups underrepresented in computing.

A second intention of e-textiles was not only to broaden participation in computing but also to raise interest in computing by providing materials such as textiles and activities such as sewing which have been associated more with feminine traditions [16]. Individual programs such as Digital Youth Divas [17] and Gwen Girls [18] have taken advantage of this intersection of gendered disciplines. In addition, these programs involving e-textiles have also been designed to draw girls specifically from underrepresented groups. The early research in computer science education provides ample evidence that girls have not been interested in computing. Work by Yardi and Bruckman found that teenagers perceived computing as "boring, solitary and irrelevant to the real-world" (p. 42) [19]. Thus, we not only need to introduce more girls and learners from underrepresented communities in computation but we also need to address how e-textiles improve their interest in and perceptions about computing.

Finally, Buechley and colleagues saw potential to study the learning of computational concepts and practices as they engage 
with e-textiles [14]. Kafai, Fields and Searle's [20] study highlighted the rich possibility of computational learning within the context of a high school e-textiles class. Students developed computational concepts such as data, conditionals, loops, etc., and practices such as testing and debugging, thinking iteratively, etc., as they made e-textiles artifacts. Learning with e-textiles not only involved computational concepts but also circuit design, a topic traditionally housed in science education. In addition, the extensive crafting involved in making e-textiles artifacts demands creative thinking as learners wrestle with embedding circuitry and coding while attending to artistic and aesthetic dimensions. Through this synthesis, we attempt to assess in which ways the promises of e-textiles to broaden participation, increase interest, and deepen learning have been realized in K-16 education.

\section{Synthesis Approach}

We conducted a meta-synthesis [10] of all the literature on etextiles published between 2007 and 2018 by focusing on searches of databases and information from colleagues working in the field. We started with systematic searches across digital libraries and conference proceedings as published in ACM Digital Library, ProQuest, EBSCO, JSTOR, and Google Scholar with keywords such as "electronic textiles," "wearables," "LilyPad Arduino" and "Circuit Playground". Furthermore, we reached out to the key contributors in the field seeking their input on our search and providing copies of publications not captured in the search.

At the conclusion of this process, our repository had 110 papers including reviews, commentaries, tool design, and evaluation studies. For the inclusion in our synthesis, we selected studies that satisfied the following criteria: (a) used e-textiles construction kits with a group of learners, either K-12 students or adults; (b) were situated in school classrooms and out-of-school settings such as after-school clubs, library workshops, or summer programs; and (c) involved examination of outcomes of learning, focusing on academic content of circuit design, computation, and/or outcomes of learner interest in and attitude towards computation. For example, a paper by Kim, Paulos, and Gross [51] that designed and prototyped an e-textile wearable without direct educational implications was not included in our synthesis. These selection criteria resulted in a final list of 46 studies. We then extracted from each paper demographic details about the learners (age, gender and ethnicity, if available); the duration, context and intention of the study; e-textile construction kits used; and the types of artifacts made by learners. We further categorized academic outcomes into the following topics: (1) interests and attitudes towards CS; (2) learning about circuits, and (3) learning about computation, ranging from basic ideas such as sequencing and variables to more advanced ideas of programming analog sensors and actuators. Some studies addressed more than one topic. We note that 43 out of the 46 studies included in our synthesis employed qualitative methods using participant observations, interviews, or student journal entries for data collection. Only three of the studies employed mixed methods and a quasi-experimental design (see [21, 22, 23]).

\section{Findings}

We present the key findings from our synthesis in the sections below, starting with a general overview of who participated, where and what participants did with e-textiles followed by a closer examination of outcomes focused on broadening participation, increasing interest, and deepening learning with etextiles.

\subsection{Engaging with E-Textiles}

Across all studies included in our synthesis, a total of more than 1,300 youth and adults participated in different kinds of e-textiles activities, with an average group size of 27 learners [23, 24, 25]. The majority of studies involved middle (44\%) and high school (40\%) youth between the ages of 12-18 years followed by elementary school students (13\%) of ages 8-11 years. Only 10 studies involved adults such as undergraduate and graduate college students $[26,27,28,29]$ as well as science and computer science teachers $[30,31,32]$ among others $[7,14,33]$.

On average, most workshops or classes were 15 hours in duration. Looking at the contexts in which these studies were conducted, more than half (56\%) took place in out-of-school spaces such as after-school classes, computer clubs, summer camps and museums [22, 34, 35] while $40 \%$ of them took place within formal learning spaces such as grade-school classrooms and universitylevel courses (e.g., [26, 36, 37]). Hackathon competitions [29], teacher professional development [31], and online platforms [38] were other rare sites of research and mostly frequented by adult participants.

What kind of e-textile artifacts did participants make with these construction kits? We identified two distinct artifacts and approaches: (1) repurposing old clothing and textile accessories and (2) sewing new textile toys and accessories. In the first category, we found that participants augmented-often their own-tote bags, jackets, hats, and shoes with new features and functionalities (see $[7,25,35]$ ). In the second category, participants sewed from scratch textile artifacts such as bracelets, bookmarks, and interactive monsters (eg., [39, 40]) using the templates developed by Qiu and colleagues [41]. The LilyPad construction kit along with text-based Arduino programming or block-based Modkit programming language were used most frequently in these designs, with only a few studies using the other commercially available Circuit Playgrounds [30] or other sewable microcontrollers such as Adafruit Flora [21].

Across both approaches, basic projects involved students stitching simple lighting circuits to backpacks or making artifacts such as wristbands and represented the most frequent e-textile designs. Slightly advanced projects often included interactive artifacts using digital inputs such as buttons and switches. More advanced projects involved analog sensors and used the sensor output to trigger certain behaviors such as playing music or making lights blink in different patterns but were far less prominent $[26,29]$. We will discuss below the ramifications of 
these project designs on learning outcomes across the field at large.

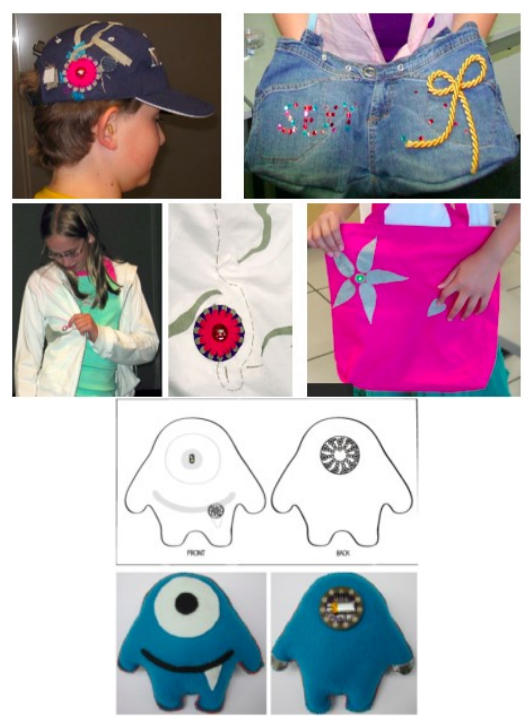

Figure 2a: Repurposing existing textiles (from left to right): hat, pants, jacket (with detail), and tote bag (drawn from $[34,25])$; Figure 2b: Sewing new projects from Buechley and Qiu's [42] Sew Electric: template (upper row), plush toy monsters (lower row)

\subsection{Broadening Participation in Computing}

Of the 46 studies included in our synthesis, more than half (56\%) focused on broadening perspectives about computing. One expectation of introducing e-textile construction kits was to make computing activities more accessible to a wider range of participants, particularly girls and women. Among the studies (only $73 \%$ of all the studies) that provided this demographic detail, $61 \%$ (567/928) of the participants were female while 39\% (361/928) were male. This proportion, surprisingly, is very similar to what Buechley and Hill [15] observed in their survey of the LilyPad Arduino projects in the wild as discussed in the background section. This large proportion of women and girls participating in computation while making with e-textiles alone is a promising finding in support of broadening participation, especially when compared to what is known about the participation of women and girls in other computing activities. For instance, only $30 \%$ of total participants in the first LEGO robotics competition were girls [43], and girls comprised of only $22 \%$ of students who took AP Computer Science classes across the nation in 2015 (CSForAll).

Another dimension of broadening participation that emerged from our review concerned the ethnicity of participants. While less than half of the studies (49\%) provided this demographic information, it is nonetheless an important factor. Close to half (48\%) of the learners came from underrepresented groups such as Hispanics/Latinos, African-Americans and American Indians, followed by $37 \%$ who identified as Caucasian and $11 \%$ who identified as Asian, Pacific Islander or mixed; $4 \%$ declined to racially identify themselves. Many e-textiles programs were targeted outreach efforts to bring underrepresented student groups into computing (eg., [17, 18]) thus explaining the large number of underrepresented participants in e-textile activities. However, we have to note that acknowledging the demographic details of the participants becomes extremely important, especially in this day and age when we want to work towards making computing education more equitable. Overall, based on the information available, we can conclude that the use of e-textile construction kits was not only successful in broadening participation by including a larger percentage of women and girls but surprisingly included also large number of participants from ethnically underrepresented groups.

\subsection{Increasing Interest in Computing}

The use of e-textile construction kits did not only attract diverse groups into computation but also increased interest in and develop positive attitudes towards computing among K-16 learners. We already noted that a majority (56\%) of the studies in our synthesis intended to broaden participants' perspectives about computing, either by providing more opportunities to be creative and express themselves or develop computing identities. Participants across all age groups-from elementary students to science teachersreported more positive attitudes towards circuitry and computing after working with e-textiles (see [13, 17, 20, 21, 39]). A few studies noted that one of the reasons why e-textiles led to such positive attitudes towards computing was related to participants' ability to customize their e-textile artifacts according to their personal and cultural interests. For instance, Fields and Kafai [35] observed middle and high school students interweave their personal interests in anime characters into e-textiles projects in school while Pinkard and colleagues [17] saw girls adopting flowers from a favorite meme to make fabric e-flowers. This finding also applied to older learners: Fields and King [27] observed adult learners integrating their knowledge from homes with e-textiles while Lovell and Buechley [39] saw older participants create personally meaningful artifacts such as bike speedometers.

Likewise, cultural interests also found room in e-textiles (e.g., $[27,32,37])$. For instance, Kutznetsov and colleagues [18] observed e-textiles artifacts to serve as "healing materials" for at-risk African American girls (ages 10-12 years) with opportunities to express themselves in creative ways while Kafai and her colleagues [20] saw Native American students design artifacts that echoed elements of their culture such as native flowers and plants in a project quilt. While the above studies pointed at possible cultural connections that increased interest among young learners, Davis and colleagues' study [21] comparing e-textiles Arduino kit with the functionally similar traditional Arduino kit in an all-girls high school observed that the traditional Arduino construction kit produced the same positive effect on girls' self-identification with computing as the e-textiles kit. Lack of clear reasons for this observation forced the researchers to hypothesize that the context in which the study was conducted, an all-girls middle school, did not allow for gender-based stereotypes to appear and influence computing learning in this context. Furthermore, they highlighted the need for further examination of the relationship between the 
gendered nature of e-textiles activity and girls' perception about computing. Our synthesis also highlighted the need for studies to further examine the nuances of interactions to tease out the elements of tool design and learning environments that provide these unique opportunities for creative expression with computing.

\subsection{Deepening Learning of Circuitry and Computing}

Studies that examined learning of academic content fell along a wide spectrum-from basic circuitry and computing ideas such as instruction sequencing to more advanced ideas of integrating multiple analog inputs and outputs. Almost a third of the 46 studies focused on learning academic content, either circuitry or computing, by making e-textiles. Out of these 16 studies, eight of them investigated learning of simple circuit concepts such as polarity and eight on basic computing concepts such as digital input/outputs and control flow. Only four studies examined more advanced programming concepts in making e-textiles such as analog inputs and outputs, data structures, and nested conditionals. Although some of the advanced projects demanded design and implementation of complex circuits and sophisticated crafting, very few studies systematically examined the influence of these domains on developing computational ideas [28]. In contrast to the previous sections that provided ample evidence of broadening participation and increasing interest in computing, learning academic content, in particular more advanced computing concepts was limited when making e-textiles.

The most compelling evidence of learning with e-textiles comes from students' understanding of circuit design. Peppler and Glosson [22] explored early on this possibility of using e-textiles to introduce circuitry concepts such as current flow, connections and polarity as students sewed on simple circuits onto their t-shirts. In this study, significant changes between pre- and post-tests clearly indicate that a class of 17 middle school students improved understanding of simple circuitry concepts after making e-textile wearables. A later quasi-experimental study by Tofel-Grehl and colleagues [23] provided additional evidence that 75 students in science classes learned significantly better about circuit design when working with e-textiles than those who learned about circuits with traditional circuitry construction kits such as breadboards and alligator clips. Using a multiple-choice assessment instrument crafted from standardized test items, researchers attributed the differences in student understanding to the nature of e-textile components that allowed for mistakes that would then lead to better learning by debugging and fixing errors. Components such as un-insulated conductive thread increased transparency since they afforded unique opportunities for students to explore concepts such as current flow and short circuits when compared to conventional circuit construction kits.

Along with studying students' learning of basic circuit design nine studies examined students' learning of simple computing concepts such as variables, instruction sequencing, and simple digital input/output. Most of these studies involved students sewing actuators such as LEDs, sometimes including input devices such as buttons and switches, and programming using simple variables, binary operators, and conditional statements to produce different light patterns (see [39, 41, 44]). For example, students employed multiple lights and buttons to make interactive wall murals that would display different lighting patterns when the user pressed definite combinations of these buttons. As noted in section 4.1, most of these student projects were limited to making artifacts such as bracelets, murals or accessories augmented with simple interaction. This need to diversifying project ideas was noted in Peppler and colleagues [50] study where they conducted an expert analysis of 166 e-textiles projects online to identify creativity in these projects only to find that even experts found that a good majority of them were mostly basic, "first LED" projects.

Only four studies reported students making artifacts that involved more advanced computational constructs such as analog inputs and outputs, arrays, and complex control-flows, integration of multiple sensors and actuators (see [26, 27, 29, 45]). For instance, in Eisenberg and colleagues' [26] semester-long seminar, undergraduate engineering students developed e-textile projects such as heating-and-cooling jacket and lighting-cum-music t-shirt that utilized a wide range of electronic components while in Richard and colleagues' [29] overnight hackathon, teams of undergraduate engineering and design students crafted wearable music players and game controllers, sensory reactive clothing for babies, and responsive purses. These e-textile projects involved computational concepts beyond conditional and event-based programming to include data processing from multiple sensors and program actuators from a wider electronics community.

\section{Discussion}

Our synthesis of introducing e-textiles during the last decade revealed not only promising outcomes but also raised several issues if we want to further computer science education with etextiles. We were heartened to see that engagement with e-textiles resulted in broadening participation and increasing interest in computing for significant numbers of youth and adults, especially from underrepresented groups. Furthermore, we identified limited efforts to explore and examine possibilities for deeper learning of more advanced computational concepts. Some studies provided examples of e-textile projects with more advanced computational concepts such as handing analog data or designing sophisticated control flows using loops and conditionals, but these were designed by undergraduate students with already significant disciplinary expertise in engineering, computation or arts. The findings of this synthesis thus provide directives for moving forward in the following directions: developing a better understanding of learning with e-textiles, creating assessments for understanding learning with e-textiles, and promoting the design of more advanced e-textile projects in K-12 CS education.

\subsection{Understanding Learning with E-Textiles}

Our synthesis demonstrated that e-textiles broadened participation in computing to include not only women but also learners from underrepresented groups. However, the exact reasons how these 
construction kits are drawing newer groups of participants into computing are unknown. While some of the studies alluded to the degree of personalization that these kits afford, there could be other reasons too. For example, Tofel-Grehl and colleagues' [23] study pointed out the different kinds of familial connections students drew on as they made e-textiles artifacts. Similarly, Searle and colleagues [37] noted how students were drawing on their familial and cultural funds of knowledge as they learned computing in classrooms. These are important connections but not yet well-understood in CS education research. A more in-depth exploration of these connections will advance our understanding of their role in developing computing identities and agency. Overall, there is a need for studies that further examine learning in these contexts to understand the features of these construction kits that enable broadening of participation and engage in learning.

\subsection{Assessing Learning with E-Textiles}

In our synthesis, we reviewed separately the multiple domains of coding or circuitry involved in learning with e-textiles, but there is a definite need to better understand the integrated or intersectional nature of problem-solving that happens as learners make and debug e-textiles projects. Many projects in computer science now promote learning integrated with other STEM subjects-just like e-textiles do-but there is little knowledge of how such integrations can be most productively facilitated for learners. For understanding learning about computational concepts and practices, we have just started developing measures that capture the intersectional nature of learning about circuitry and computing with e-textiles [44]. However, we know that crafting and aesthetics are also intertwined in the context of learning with e-textiles [20, 28]. We should capture learning outcomes that provide a more holistic assessment, not privileging one academic domain over the other. One possible approach is through portfolios that ask students to document and reflect upon their learning with e-textiles [46, 47]. Here learners can describe their artifacts with photos, annotated blueprints of circuit diagrams and Arduino code as a part of documenting their process and practices, moving the focus away from a final product. Furthermore, they can also describe challenges they had to overcome [46] which have proven to be insightful in understanding student gains in computational concepts, practices and perspectives [48]. Nevertheless, we see a need to push development of different assessments formats that allow us to capture the complexities of learning about computation with etextiles.

\subsection{Developing Advanced E-Textiles Projects}

Our synthesis also identified the critical need to develop more complex e-textile activities that raise the ceiling of what students can learn about computing. We have a few examples from undergraduate classes and hackathons [26, 28, 29] where learning with e-textile projects expanded by bringing together an eclectic set of hardware such as fans, solar panels, fabric insulators, electroluminescent films, MP3 players with other microcontroller boards. These examples suggest that the introductory toolkit needs to include a wider variety of input and output devices so that novices can explore a more diverse set of computational ideas. To introduce such projects to $\mathrm{K}-12$ students will also require careful consideration and design of scaffolds.

When e-textile construction kits were introduced over a decade ago they offered to broaden participation and increase interest in computing. While some of these promises have been realized there is a need to design tools, activities and pathways that provide opportunities for learners to explore a wider range of computational concepts, thereby enabling deeper learning. The promise of e-textiles to expand the landscape of computer science education to broaden participation and increase interest among learners from underrepresented groups will only gain strength with more research to foster deeper computational learning and assessment.

\section{ACKNOWLEDGMENTS}

This work was supported by the National Science Foundation grant to Yasmin Kafai, Jane Margolis, and Joanna Goode (\#1509245/1512760/1510725). Any opinions, findings, conclusions or recommendations expressed in this paper are those of the authors and do not necessarily reflect the views of either NSF or the University of Pennsylvania. Special thanks to Deborah Fields, Debora Lui, Justice T. Walker, and Mia Shaw for their valuable feedback.

\section{REFERENCES}

[1] Jane Margolis and Rachel Estrella. 2017. Stuck in the shallow end: education, race, and computing, The MIT Press, Cambridge, MA, USA

[2] Jeff Stern, Emily Reid, and Kari Bancroft. 2015. Teaching introductory computer science for a diverse student body: Girls who code style. In Proceedings of the 46th ACM Technical Symposium on Computer Science Education, ACM, 2015, 705-705.

[3] Yasmin B. Kafai, Kylie A. Peppler, and Robbin N. Chapman. 2009. The Computer Clubhouse: Constructionism and Creativity in Youth Communities. Technology, Education--Connections. Teachers College Press. 1234 Amsterdam Avenue, New York, NY, USA.

[4] Mitchel Resnick, John Maloney, Andrés Monroy-Hernández, Natalie Rusk, Evelyn Eastmond, Karen Brennan, Amon Millner, ... and Yasmin B. Kafai. 2009 Scratch: programming for all. Communications of the ACM 52, 11 (2009), 60-67.

[5] Caitlin Kelleher and Randy Pausch. 2005. Lowering the barriers to programming: A taxonomy of programming environments and languages for novice programmers. ACM Computing Surveys (CSUR) 37, no. 2 (2005): 83-137.

[6] Jane Margolis and Joanna Goode. 2016. Ten lessons for computer science for all. ACM Inroads 7, 4 (2016), 52-56.

[7] Leah Buechley. 2006. A construction kit for electronic textiles. In Wearable Computers, 10th IEEE International Symposium 2006, 83-90.

[8] Leah Buechley, Kylie Peppler, Michael Eisenberg, and Kafai Yasmin. 2013. Textile Messages: Dispatches from the World of E-Textiles and Education. New Literacies and Digital Epistemologies. Volume 62. Peter Lang Publishing Group. 29 Broadway 18th Floor, New York, NY 10006, 2013.

[9] Mark Guzdial. 2015. Learner-centered design of computing education: Research on computing for everyone. Synthesis Lectures on Human-Centered Informatics 8, 6 (2015), 1-165.

[10] Harsh Suri and David Clarke. 2009. Advancements in research synthesis methods: From a methodologically inclusive perspective. Review of Educational Research 79, 1 (2009), 395-430.

[11] Harsh Suri. 2013. Epistemological pluralism in research synthesis methods. International journal of qualitative studies in education 26, 7 (2013), 889-911.

[12] Maggie Orth, Rehmi Post, and Emily Cooper. 1998. Fabric computing interfaces. In CHI 98 conference summary on Human factors in computing systems, ACM, 1998, 331-332.

[13] Grace Ngai, Stephen CF Chan, Joey CY Cheung, and Winnie WY Lau. 2009. The TeeBoard: an education-friendly construction platform for e-textiles and wearable computing. In Proceedings of the SIGCHI Conference on Human Factors in Computing Systems, ACM, 2009, 249-258. 
[14] Leah Buechley, Nwanua Elumeze, and Michael Eisenberg. 2006 Electronic/computational textiles and children's crafts. In Proceedings of the 2006 conference on Interaction design and children, ACM, 2006, 49-56.

[15] Leah Buechley and Benjamin M. Hill. 2010. LilyPad in the wild: how hardware's long tail is supporting new engineering and design communities. In Proceedings of the 8th ACM conference on designing interactive systems, ACM, 2010, 199-207.

[16] Leah Buechley and Michael Eisenberg. 2008. The LilyPad Arduino: Toward wearable engineering for everyone. IEEE Pervasive Computing 7, 2 (2008), 12-15.

[17] Nichole Pinkard, Sheena Erete, Caitlin K. Martin, and Maxine McKinney de Royston. 2017. Digital Youth Divas: Exploring narrative-driven curriculum to spark middle school girls' interest in computational activities. Fournal of the Learning Sciences 26, 3 (2017), 477-516.

[18] S. Kutznetsov, L. Trutoiu, C. Kute, I. Howley, D. Siewiorek, and E. Paulos. 2011. Breaking Boundaries: Mentoring with Wearable Computing. In Proceedings of CHI 2011, 2957-2966.

[19] Sarita Yardi and Amy Bruckman. 2007. What is computing?: bridging the gap between teenagers' perceptions and graduate students' experiences. In Proceedings of the third International workshop on Computing Education Research, ACM, 2007, 39-50.

[20] Yasmin B. Kafai, Deborah Fields, and Kristin Searle. 2014. Electronic textiles as disruptive designs: Supporting and challenging maker activities in schools. Harvard Educational Review 84, no. 4 (2014): 532-556.

[21] Richard L. Davis, Christopher Proctor, Michelle Friend, and Paulo Blikstein. 2018. Solder and Wire or Needle and Thread: Examining the Effects of Electronic Textile Construction Kits on Girls' Attitudes Towards Computing and Arts. In Proceedings of the International Conference of Learning Sciences, 2018, 800-808.

[22] Kylie Peppler and Diane Glosson. 2013. Stitching circuits: Learning about circuitry through e-textile materials. Fournal of Science Education and Technology 22, 5 (2013), 751-763.

[23] Colby Tofel-Grehl, Deborah Fields, Kristin Searle, Cathy Maahs-Fladung, David Feldon, Grace Gu, and Chongning Sun. 2017. Electrifying engagement in middle school science class: improving student interest through e-textiles. Fournal of Science Education and Technology 26, 4 (2017), 406-417.

[24] Yasmin B. Kafai and Kylie A. Peppler. 2014. Transparency Reconsidered: Creative, Critical, and Connected Making with E-textiles. DIY citizenship: Critical making and social media (2014), 179-188.

[25] Eva-Sophie Katterfeldt, Nadine Dittert, and Heidi Schelhowe. 2009. EduWear: smart textiles as ways of relating computing technology to everyday life. In Proceedings of the 8th International Conference on Interaction Design and Children, ACM, 2009, 9-17.

[26] Michael Eisenberg, Ann Eisenberg, and Yingdan Huang. 2013. Bringing e-textiles into engineering education. Textile Messages: Dispatches from the world of etextiles and education (2013), 121-129.

[27] Deborah A. Fields and Whitney L. King. 2014. So, I think I'm a programmer now. Developing connected learning for adults in a university craft technologies course. In Learning and Becoming in Practice: The International Conference of the Learning Sciences (ICLS), 1, 927-936.

[28] Victor R. Lee and Deborah A. Fields. 2017. A rubric for describing competences in the areas of circuitry, computation, and crafting after a course using e-textiles. The International fournal of Information and Learning Technology 34, 5 (2017), 372-384.

[29] Gabriela T. Richard, Yasmin B. Kafai, Barrie Adleberg, and Orkan Telhan. 2015, StitchFest: Diversifying a College Hackathon to broaden participation and perceptions in computing. In Proceedings of the 46th ACM Technical Symposium on Computer Science Education, ACM, 2015, 114-119.

[30] Deborah A. Fields, Debora Lui, and Yasmin B. Kafai. 2017. Teaching computational thinking with electronic textiles: High school teachers' contextualizing strategies in exploring computer science. Siu-cheung KONG The Education University of Hong Kong, Hong Kong (2017), 67-72.

[31] Kristin A. Searle, Colby Tofel-Grehl, and Vicki Allan. 2016. The E-Textiles Bracelet Hack: Bringing Making to Middle School Classrooms. In Proceedings of the 6th Annual Conference on Creativity and Fabrication in Education, ACM, 2016, 107-110.

[32] Colby Tofel-Grehl and Kristin Searle. 2017. Critical Reflections on Teacher Conceptions of Race as Related to the Effectiveness of Science Learning. Fournal of Multicultural Affairs 2, 1 (2017), 4.

[33] Leah Buechley, Mike Eisenberg, and Nwanua Elumeze. 2007. Towards a curriculum for electronic textiles in the high school classroom. In ACM SIGCSE Bulletin, 39 (3), 28-32.
[34] Leah Buechley, Mike Eisenberg, Jaime Catchen, and Ali Crockett. 2008. The LilyPad Arduino: using computational textiles to investigate engagement, aesthetics, and diversity in computer science education. In Proceedings of the SIGCHI conference on Human Factors in Computing Systems, ACM, 2008, 423-432.

[35] Deborah A. Fields and Y. B. Kafai. 2011. Crafting Identities: E-Textile Artifacts as Mediators in High Tech Communities. In H. Spada, G. Stahl (Eds), N. Miyake, \& N. Law (Eds), Connecting Computer Supported Collaborative Learning to policy and practices: CSCL '11 community events proceedings, 2, Short Papers \& Posters, International Society of the Learning Sciences, 910- 911.

[36] Deborah A. Fields, Yasmin B. Kafai, Tomoko Nakajima, and Joanna Goode. "Teaching Practices for Making E-Textiles in High School Computing Classrooms." In Proceedings of the 7th Annual Conference on Creativity and Fabrication in Education, p. 5. ACM, 2017.

[37] Kristin A. Searle and Yasmin B. Kafai. 2015. Boys' Needlework: Understanding Gendered and Indigenous Perspectives on Computing and Crafting with Electronic Textiles. In International Computing Education Research, 2015, 31-39.

[38] Emily Lovell and Leah Buechley. 2011. LilyPond: an online community for sharing e-textile projects. In Proceedings of the 8th ACM Conference on Creativity and Cognition, ACM, 2011, 365-366.

[39] Debora Lui, Breanne K. Litts, Sari Widman, Justice T. Walker, and Yasmin B. Kafai. 2016. Collaborative Maker Activities in the Classroom: Case Studies of High School Student Pairs' Interactions in Designing Electronic Textiles. In Proceedings of the 6th Annual Conference on Creativity and Fabrication in Education, ACM, 2016, 74-77.

[40] Anne Weibert, Andrea Marshall, Konstantin Aal, Kai Schubert, and Jennifer Rode 2014. Sewing interest in E-textiles: analyzing making from a gendered perspective. In Proceedings of the 2014 conference on Designing Interactive Systems, ACM, 2014, 15-24.

[41] Kanjun Oiu, Leah Buechley, Edward Baafi, and Wendy Dubow. 2013. A curriculum for teaching computer science through computational textiles. In Proceedings of the 12th International Conference on Interaction Design and Children, ACM, 2013, 20-27.

[42] Leah Buechley and Kanjun Qiu. 2014. Sew electric.

[43] Alan Melchior, Tracy Cutter, and Faye Cohen. 2004. Evaluation of FIRST LEGO league. Waltham, MA: Center for Youth and Communities, Heller Graduate School, Brandeis University.

[44] Breanne K. Litts, Yasmin B. Kafai, Kristin A. Searle, and Emily Dieckmeyer. 2016 Perceptions of Productive Failure in Design Projects: High School Students' Challenges in Making Electronic Textiles. In Proceedings of International Conference of Learning Sciences, 2016.

[45] Orkan Telhan, Yasmin B. Kafai, and Breanne K. Litts. 2016. Designing for Connected Making: Supports for Collaboration and Community building in Crafting Activities. Makeology Volume 1: Makerspaces as Learning Environments (2016), 223-236

[46] Debora Lui, Gayithri Jayathirtha, Deborah A. Fields, Mia Shaw, and Yasmin Kafai. 2018. Design Considerations for Capturing Computational Thinking Practices in High School Students' Electronic Textile Portfolios. In Proceedings of International Conference of Learning Sciences, 2018, 721-728.

[47] Owen Astrachan and Amy Briggs. 2012. The CS principles project. ACM Inroads 3, no. 2, 38-42.

[48] Yasmin B. Kafai, Kristin Searle, Crîstobal Martinez, and Bryan Brayboy. 2014 Ethnocomputing with electronic textiles: culturally responsive open design to broaden participation in computing in American Indian youth and communities. In Proceedings of the 45th ACM Technical Symposium on Computer Science Education, ACM, 2014, 241-246.

[49] Gayithri Jayathirtha, Deborah A. Fields, and Yasmin B. Kafai. 2018. Computational concepts, practices, and collaboration in high school students' debugging electronic textile projects." In Conference Proceedings of International Conference on Computational Thinking Education 2018. 27-32.

[50] Kylie Peppler, Diane Glosson, Yasmin Kafai, Deborah Fields, and Kristin Searle. 2011. Articulating creativity in a new domain: expert insights from the field of $\mathrm{e}$ textiles. In Proceedings of the 8th ACM conference on Creativity and Cognition, ACM, 2011, 385-386.

[51] Sunyoung Kim, Eric Paulos, and Mark D. Gross. 2010. WearAir: expressive tshirts for air quality sensing." In Proceedings of the fourth International Conference on Tangible, Embedded, and Embodied Interaction, ACM, 2010, 295-296. 